Cite as:

Pieczka, M. and Casteltrione, I. 2019. AlcoLOLs, Re-thinking Drinking: Developing a shared leadership approach for alcohol education. Health Education Journal. Forthcoming.

\title{
AlcoLOLs, Re-thinking Drinking: Developing a shared leadership approach for alcohol education
}

Magda Pieczka and Isidoropaolo Casteltrione

Division of Media, Communications and Performing Arts, Queen Margaret University Edinburgh, Musselburgh, Scotland UK

\begin{abstract}
Objective: The aim of this article is to extend and elaborate ways of conceptualising, enabling and practising peer leadership in whole-school alcohol education programmes.

Design: Qualitative study involving individual and group interviews.

Setting: The AlcoLOLs project took place in six secondary schools in North East Edinburgh (Scotland) from 2013 to 2015.

Methods: 21 individual and 4 group interviews with young people aged 14-18 who acted as peer leaders in the AlcoLOLs project. Interviews were conducted throughout the duration of the project as a means of hearing peer leaders' individual voices, monitoring progress and evaluating the intervention. Data were analysed using the principles of thematic analysis.

Results: The intervention demonstrates transformative multilevel learning (i.e. cognition, civic/communal attitudes, self-identity, self-efficacy, specific communication/team skills) for peer leaders resulting from the shared leadership process. Results indicate that there is an element of continuity between antecedents, process and outcomes of shared leadership which, in the context of peer education, needs to be seen as an iterative rather than a linear process. Drawing on these findings, a model for a whole-school alcohol peer education intervention is developed. The model is underpinned by critical dialogic principles and reframes alcohol consumption as action rather than behaviour.

Conclusion: This article redefines peer leadership in alcohol education interventions for young people as a process involving formal, informal, individual, and shared leadership. Combined with a whole-school dialogic intervention, this approach can lead to the development of alcohol consumption/abstinence as a practice that focuses on articulation of a self-identity drawing on both individual/personal and civic aspects.
\end{abstract}

\section{Keywords}

Alcohol education; peer education; shared leadership; dialogue; 


\section{Introduction}

Alcohol consumption has been falling in Scotland and in the UK as a whole since its peak in 2004 , in response to wide-ranging policy efforts implemented in the last few years across the country (Institute of Alcohol Studies, 2018; NHS Health Scotland, 2016; Smith and Foxcroft, 2009). Recognising the problem of harmful alcohol consumption, governments in charge of public health within the devolved UK acted to control the availability of alcohol (licensing), its marketing, affordability - with Scotland in particular regulating the minimum price of alcohol through its newly implemented Alcohol (Minimum Pricing) (Scotland) Act 2012 - and changing attitudes and behaviours through health interventions, including education (Public Health England, 2016; Gray and Leyland, 2017).

Alcohol consumption by young people (aged 16-24), a particular concern in Scotland (Scottish Executive, 2002), has fallen more than for other age groups, although young drinkers now tend to binge drink ${ }^{1}$ more than older drinkers (Office for National Statistics, 2017). A report from Demos on youth drinking (Wyborn, 2016) draws attention to the emerging understanding of the complex interplay of factors behind this new trend. While giving credit to health education, Wyborn also points to diverse factors at play, such as the less well acknowledged role of changing social norms privileging healthy lifestyle; approbation of drinking circulating through social media and peer group cultures; young people's lack of interest in long-term effects of alcohol; and poor data on the availability of alcohol for the youngest people (aged 16-17) covered by the national statistics.

It is in this context that we offer an analysis of a three-year long educational intervention conducted in six secondary schools in Edinburgh (2013-2015). The AlcoLOLs, as the project was named by its young participants, combined peer education with a dialogic approach in order to develop a health education intervention that looked beyond the paradigm of individual learning. Dialogue is a form of discourse that emphasises listening and inquiry to foster mutual respect and understanding (Broome 2009). It is about building relationships, co-producing understanding of the issues through the suspension of reciprocal assumptions and a quest for common ground (for a detailed discussion of dialogue see Pieczka et al., 2010, and Pieczka and Wood, 2013). Our approach utilised dialogue as a method of both inquiry and will formation (Pieczka, 2011). Focusing on collective capacity to generate knowledge, the intervention aimed to help teenagers realise that they do not need to make isolated judgments about alcohol consumption governed by their understanding of social norms. Instead, shared critical reflection enabled them to reframe alcohol drinking and to develop new ways of conducting themselves in relation to alcohol.

In order to add to our understanding of both the actual educative process and the social dynamics involved in peer education, this article re-interprets the intervention as a process of shared leadership. It proposes shared peer leadership as a model for alcohol education in whole-school interventions (Pieczka et al., 2016; Pieczka and Wood, 2013), drawing on the emerging collectivist leadership paradigm in management studies (Yammarino et al., 2012; Cullen-Lester and Yammarino, 2016b).

\footnotetext{
${ }^{1}$ Binge drinking or Heavy Episodic Drinking (HED) is defined by World Health Organization (2019) as drinking at least 60 grams or more of pure alcohol on at least one occasions in the past 30 days
} 


\section{Peer education and peer leadership}

Approaches to alcohol education in the $21^{\text {st }}$ century have moved beyond focusing on knowledge deficits, personality, affect, and social skills to recognise now a broader range of environmental factors such as: parental influence, curriculum, links with health and community services, social, physical and cultural settings Lee et al., 2016). Recent literature looking at educational interventions tackling youth drinking stressed the importance of recognising the social nature of drinking, and the impact of peer group cultures and relationships. (de Visser et al., 2015; de Visser et al., 2013; Livingstone et al., 2011; Percy et al., 2011). According to de Visser at al. (2015), educational interventions can combine social marketing, as the logic behind the individual-level influence sought across targeted populations, with resilience-based approaches that treat young non-drinkers/ moderate drinkers as "experts" in responsible alcohol consumption. In this context, peer education is seen as useful for a number of reasons: it uses credible communicators (peers) thus increasing the persuasiveness; it is able to connect knowledge, motivation, and skills through peer educators' experiences; and it aligns with a resilience framework by 'developing not only the individual capacities but also broader protective mechanisms' (de Visser et al., 2015: 351).

Despite being a well-established and internationally promoted approach to health education, peer education remains poorly understood (Southgate and Aggleton, 2017). On the whole, peer education is accepted as beneficial (Harden et al., 2001), with evidence showing a range of effects on peer leaders' knowledge, attitudes and behaviours that fit well with both Information-Motivation-Behavioural Skills (IMB) model of health behaviour (Fisher et al., 2003) and resilience frameworks, for example: self-confidence, self-esteem, general motivation, increased knowledge, or communication skills and knowledge and behaviour of participants (Ochieng, 2003; see also Foxcroft and Tsertsvadze, 2011). The effects of peer education have, however, shown to be uneven (Borgia et al., 2005; Sacks-Davis et al.., 2012; Tolli, 2012; Webel et al., 2010). Research indicates that the success of the peer-led programme hinges on the similarity between the peer educator and the recipient of health messages; the content and amount of sessions and follow-up or booster sessions; levels of student interaction; whether teachers are present during peer-led delivery of material (Shiner, 1999; Cuijpers, 2002; McDonald, 2004); and cultural similarity (Mason-Jones et al., 2011).

This rather narrow focus on the effects produced can be seen as the technicisation of peer education, i.e. the view of peer education as an input-output dynamic, the first of three major shortcomings in current understandings identified by Southgate and Aggleton (2017: 3-4). The second weaknesses is 'black boxing' of the actual educative processes of peer education, i.e. the lack of robust data and analysis of peer education in situ; what the peer educators do and 'how the process is experienced by those being educated' (Southgate and Aggleton, 2017: 4). Finally, they argue that social factors (ethics, power, embodiment and tacit knowledge) have not received sufficient attention either. This is where they locate the so far poorly explained variability of peer education's effectiveness. Our work contributes empirical material relevant to these gaps in the current knowledge of peer education. 
Peer leadership appears in health education as an element of peer education, but the exact meaning and relationship of these terms is unclear. Such ambiguity has so far been unrecognised (Velleman, 2009: 31) or set aside as unimportant,

...peer counsellor, peer tutor; or peer leader ... these terms are more similar than different. They all involve peers informing, educating, facilitating and supporting their peer groups. (Ochieng, 2003: 62).

However, a number of questions arise here, demonstrating the need to examine the meaning and relationship between concepts such as leadership and education, and activities such as informing, facilitating, leading, educating, motivating and counselling. For example: Are peer educators by default peer leaders, or can the two be separated? Do peer leaders deliver motivation by consciously wielding particular skills, or does motivation reside in the phenomenon of 'peerness'? If being a peer is defined as having similar social status or shared group membership (McKeganey, 2000) and 'similar characteristics and experiences' (de Vreede et al., 2014: 39), then to what extent is the similarity defined in objective ways by recognised classification systems (e.g., a binge drinker), and to what extent might it depend on subjective recognition of similarity, i.e. on identification? Is peer leadership defined by technical tasks such as informing or facilitating; or it is bigger than that-performance of being with others in particular (educational) contexts that inspires recognition and aspiration: 'I am like you, I like you, I want to be more like you'? Is it about conforming, or transforming? Taking our cue from Southgate and Aggleton's (2017) argument for the need to inquire more closely into the complex dynamics of peer education, we focus on peer leadership as a way forward.

\section{Shared leadership}

Research has traditionally treated leadership as 'a leader-follower interaction process' (Yammarino et al., 2012: 383). The changing realities and practices of organisational life in recent decades resulted in a paradigm shift within the field of leadership research towards the view of leadership 'as a property of the collective, not the individual' (Cullen-Lester and Yammarino, 2016b: 173). For the purpose of developing a leadership-based model of peer education, this article focuses on shared leadership, one out of several emerging collectivistic approaches in the field of leadership (Cullen-Lester and Yammarino, 2016a). Shared leadership may be defined as 'a dynamic, interactive influence process among individuals in groups for which the objective is to lead one another to the achievement of group or organizational goals or both' (Pearce and Conger, 2003: 1). To develop a leadership-based model for all-school alcohol education interventions we rely on leadership research and theory in a number of ways.

First, for analytical purposes, we retain the three-part approach to leadership as a process understood to be influenced by its antecedents and producing effects on individual followers or on collectives such as groups, organisation or networks (Yammarino et al., 2012). Second, we retain the traditional distinction between formal and informal leadership, where the first is associated with the existence of formal organisations (for example, a school) and its hierarchy of authority and power, while the latter refers to personal connections or networks that are independent of formal organisational structures (White et al., 2016). Third, we define 
shared leadership as a sustained group approach to goal attainment, characterised by mutual and shared responsibility, task interdependence and team empowerment (Yammarino et al., 2012: 396).

The core of the intervention discussed below is built around a model of shared leadership based on work of Carson et al. (2007), Serban and Roberts (2016) and Yammarino et al. (2012), summarised in Figure 1.

Figure 1: Conceptual model of shared leadership, based on Serban and Roberts (2016) and Yammarino et al. (2012)

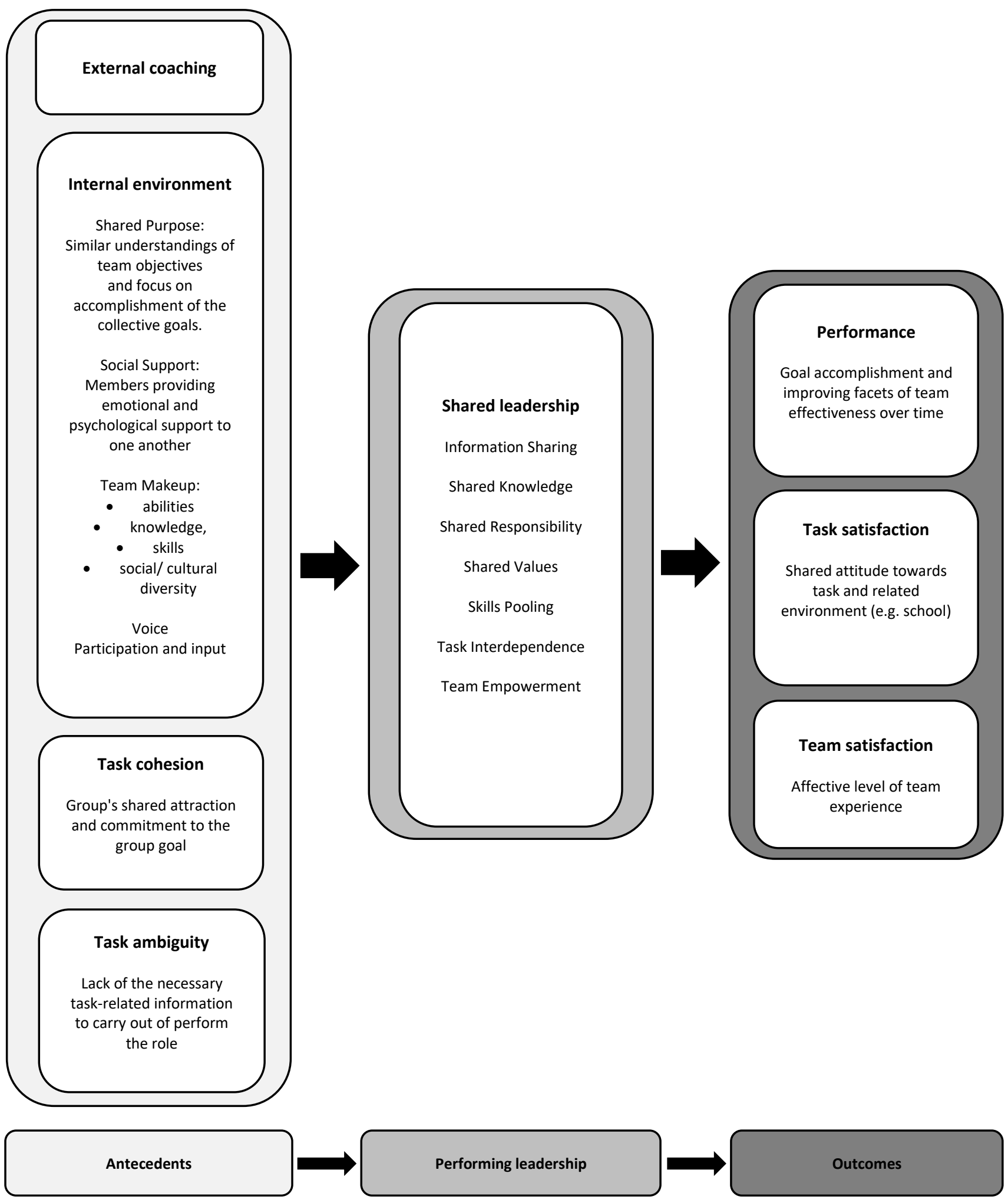




\section{The intervention - The AlcoLOLs project}

The AlcoLOLs project aimed to tackle the issues alcohol presents for young people through pupil-run in-school dialogue groups involving, typically, 12-15 participants and a team of three to four peer leaders. As much variety in terms of age, gender and ethnicity as was practically possible was designed into each group because dialogue is powered by difference, not by consensus (Wierzbicka, 2006). The project was co-designed by Portobello High School pupils along with academics at Queen Margaret University. It was piloted in Portobello High School in 2011-2012 and funded by the Robertson Trust to run in six Edinburgh secondary schools from 2013 to 2015. During this timeframe, it trained 200 dialogue facilitators, i.e. peer leaders who ran approximately 600 dialogue sessions in their respective schools, involving 3000 pupils across North East Edinburgh (for a more detailed discussion of the design, development, implementation and outcomes of the intervention see Pieczka and Wood, 2013; Pieczka et al., 2016).

The dialogue sessions were designed to: problematise alcohol; question participants' attitudes and behaviours; offer knowledge regarded by participants as useful in their routine encounters with alcohol through either their own or peer/friendship group's drinking practices; develop new communication skills to support learning and resilience; and, where appropriate, to change behaviours. The keystone of this process was the creation of a safe space, with no adults in the room to take control of the conversation. The AlcoLOLs project treated alcohol consumption as a social and cultural practice and was developed from the premise that persuasion and information-giving were insufficient communication methods to tackle the issue. Combining insights from dialogue, peer education, and a harm reduction approach, the intervention delivered a range of beneficial outcomes for participants: new skills and knowledge, change of attitudes and behaviours producing effective self-regulation of drinking, and the promise of a potentially larger-scale cultural transformation (Pieczka et al. 2016).

\section{Data collection}

Data for this article derive from 21 individual interviews and 4 group interviews with AlcoLOLs peer leaders ${ }^{2}$ drawn from a larger set of both qualitative and quantitative data gathered for the purposes of project evaluation (Pieczka et al., 2016). ${ }^{3}$ With the aim of proposing shared peer leadership as a model for alcohol education, we decided to focus on peer leaders' voices and experiences and excluded other data. Participants were recruited on voluntary basis from the pool of peer leaders. All interviews were conducted by the two academics responsible for the project as a means of hearing facilitators' individual voices, monitoring progress and evaluating the project. They were carried at the end of each year of the

\footnotetext{
2 For clarity we use the term 'peer leaders' to refer to pupils in the schools participating in the AlcoLOLs project who: took part in the training, volunteered to run dialogue groups in their respective schools, including scheduling facilitating, and modifying elements of the intervention following discussion and agreement with the two academics responsible for the project.

${ }^{3}$ Qualitative data were generated through interviews conducted with peer leaders, teachers, community and police officers, while quantitative data were obtained via evaluation questionnaires completed by pupils at the end of the dialogue sessions and benchmark questionnaires distributed to whole school populations in the three biggest schools.
} 
intervention (2013-2015), lasted between 20 and 45 minutes, and were audio-recorded. A semi-structured approach was followed, and an interview guide developed. The key areas explored in the interviews were: motivation for project involvement; experience running the project; the process of involvement from training to running dialogue groups; attitudes, behaviours and skills relevant to drinking/abstinence; sharing and transferring of new learning developed through the project across social networks, school, family and community.

The choice of combining individual and group interviews was dictated by practical reasons, in order to maximise the number of interviewees. Combining individual and group interviews for pragmatic reasons is not uncommon (Lambert and Loiselle, 2008). Such a practice may impact negatively on the trustworthiness of the findings, particularly if data sets are assumed to be equivalent and methodological underpinnings are overlooked (Barbour, 1998; Tobin and Begley, 2004).) While this needs to be recognised as a limitation of our approach, the practicalities of, and the consequent importance of flexibility in real-word research have to be acknowledged (Robson, 2011). Furthermore, there was a high degree of consistency between the themes and findings obtained through the two methods, in line with other studies which found that, overall, interview and focus group data can generate similar concepts or conclusions (Namey et al. 2016; Stokes and Bergin, 2006).

\section{Data analysis}

Data were analysed through NVivo using the principles of thematic analysis. More specifically, a combination of inductive and theoretical thematic analysis (Braun and Clark, 2006) was adopted. Initial coding captured the material related to the performance of shared leadership, while the subsequent coding phase was theoretically informed by the constructs of shared leadership identified in the literature (see Figure 1). The analysis was executed through a three-step procedure by the two authors of this article. The process was systematic, sequential, verifiable and continuous (Denzin and Lincoln, 2005). The codebook was developed through a process that combined initial inductive coding subsequently refined and informed by conceptual frameworks (Carson et al., 2007; Serban and Roberts, 2016; Yammarino et al., 2012). The next step of the analysis consisted of an iterative process of coding, discussions, and further refinements to codes. The final process of coding led to the identification of emergent themes that, in turn, informed the proposed model for a wholeschool alcohol peer education intervention.

\section{Results and discussion - The AlcoLOLs Project: Shared leadership model of peer education}

The overarching aim of the present article is to extend and elaborate ways of conceptualising, enabling and practising peer leaderships in whole-school alcohol education programmes. A model of alcohol peer education as shared leadership was developed from themes (see Table 1 ) identified in the analytic process described in the previous section. 
Table 1. Thematic analysis: Identified themes

\begin{tabular}{|c|c|}
\hline Clusters & Themes \\
\hline \multirow[t]{10}{*}{ Antecedents } & External coaching \\
\hline & Internal environment \\
\hline & Social support \\
\hline & Team make-up \\
\hline & Various abilities, knowledge and skills \\
\hline & A varied group \\
\hline & Voice \\
\hline & Task ambiguity \\
\hline & Task cohesion and shared purpose \\
\hline & Contributing to Scotland's future \\
\hline \multirow[t]{10}{*}{ Performing leadership } & Adapting and personalising the intervention \\
\hline & Information sharing and shared knowledge \\
\hline & Communicating and sharing with other AlcoLOLs \\
\hline & Sharing stories and experiences with participants \\
\hline & $\begin{array}{l}\text { Skills pooling, task-interdependence, shared responsibility and } \\
\text { distributed power }\end{array}$ \\
\hline & Shared values \\
\hline & Skills development \\
\hline & Sustained effort \\
\hline & Team empowerment \\
\hline & Helping younger pupils and others \\
\hline \multirow[t]{11}{*}{ Outcomes } & On AlcoLOLs (individual level) \\
\hline & Alcohol behaviour \\
\hline & Alcohol praxis (critical) \\
\hline & Confidence, public speaking, organisation skills and more \\
\hline & On Team \\
\hline & Improvement of performance \\
\hline & Task satisfaction \\
\hline & Team satisfaction \\
\hline & On parents \\
\hline & Positive impact on participants \\
\hline & Impacting on school as social system \\
\hline
\end{tabular}

\section{Antecedents}

In the management literature, shared leadership is seen as stemming from a combination of antecedents to do with team- and task-related characteristics (see Figure 1), and external coaching as a way of intervening in these characteristics. The starting point for the AlcoLOLs intervention, however, was the creation of the shared leadership antecedents themselves. We based this work on the dialogic approach defined by its participatory orientation and extended epistemology (adding relational, critical, and practical knowledge to the propositional knowledge that underpins public health education; for more see Pieczka and 
Wood, 2013: 165-166). The foundations for shared leadership were laid in the process of training that also modelled dialogic communication for the peer leaders and prepared them to facilitate dialogue groups using cues such as specially created short videos, storytelling, and factual knowledge about the impact alcohol has on the human body, on individuals, families and the society. This was a long process, taking around five months, involving four dialogue sessions and a big training day for leaders from all schools in the project.

"[The training day] was like a stepping stone for the group to form, and then moving on to [running the groups]." (Group interview TA) ${ }^{4}$

Thereafter, there were regular debriefs, top-up training sessions and changes to the content or organisation of the dialogue sessions themselves suggested by the peer leaders on the basis of their facilitation experiences. The desired antecedents of shared leadership, i.e. Shared Purpose, Social Support, Task Cohesion, and Voice, emerged naturally from the training process built around dialogic principles and practices. Given the definition of shared purpose and task cohesion (see Figure 1), it is perhaps unsurprising that they appeared in our data as inextricably linked. The AlcoLOLs peer leaders felt their mission was twofold: a societal one, to shape Scotland's future by changing its drinking culture, and a more school-based and generational one, of helping younger pupils to make informed decisions about alcohol.

"We are trying to shape Scotland's future and it's important for us as facilitators to know that this is what we are trying to do; it means a lot feeling part of it and knowing that we are doing something to help" (Participant 1 TVA)

"It is about mentoring the younger ones and helping them" (Participant $6 \mathrm{MH}$ )

Social Support manifested itself in the form of sharing information with other team members or encouraging and reassuring them, and, in the context of our intervention, enabled effective performance of shared leadership, the formation of friendship bonds and, in some cases, a collective identity.

"I missed the main training day so I thought I'd be really bad at it, but ... all my peers were so good they told me things so by the second week I felt I was at the same level as them..." (Participant $7 \mathrm{MH}$ )

“Others don't really understand it [being an AlcoLOL]... we became very good friends over it. It is fun to lead the groups because these are people you enjoy being around and you have a laugh about it (Participant 8 TVA)

Task Ambiguity was approached through coaching: peer leaders needed sufficient skills and support to be able to start organising and running dialogue groups at school; at the same time, there needed to be an element of creativity and improvisation left in the design to encourage group dialogue. As the intervention moved beyond the training stage, peer leaders quickly learned that sessions needed to be adapted: they used their own personal

\footnotetext{
${ }^{4}$ Letters denote interviewees' school affiliation: Meadows High School (MH), Tree Valley Academy (TVA), and Sunny Fields High School (SFH). Schools' names have been replaced with pseudonyms to protect participants' anonymity and confidentiality.
} 
experiences in order to respond to group participants' needs. This juxtaposition of certainty and creativity, which is arguably an expression of Voice, can be seen as contributing to the performance of shared leadership.

"By the end of [training] you became confident that you knew how you would run the group." (Participant $2 \mathrm{MH}$ )

"[To run the groups] you realise that you will have to adapt ..." (Group interview SFH)

"It is good because it is not all the same... [There is no] script in a way... people say different things every single time, even the facilitators" (Participant 6 TVA)

Voice is about participation and input (Carson et al., 2007). 'Under a high level of voice, team members should engage in shared leadership by being committed to and actively involved in achieving the team goals by strengthening a shared sense of direction and positive interpersonal support' (Serban and Roberts, 2016: 184). In our data, Voice was not only an antecedent 'injected' into peer leaders through training, as shown in the first quote below, but also a feature of shared leadership that developed throughout the project. Peer leaders stressed the importance of being proactive (ability to read groups and to know when to intervene) and taking ownership of the project when running the sessions. They felt that sharing their own experiences was crucial to successful dialogue sessions as it enabled the formation of a safe space in which participants, in turn, felt encouraged to share their stories and experiences.

"[The training was] very different to what I expected, I thought we'd be lectured at about alcohol. I liked how we got to add our input and talk about it ourselves. When I left I was like 'Oh, I want to go back, when's the next time?'” (Participant 2 SFH)

"If it was just talking about alcohol and what it does, the facts, it wouldn't go in or have an effect. People get more interested when they can put in their own opinions, their own take on it." (Group interview TVA)

\section{Performing shared leadership}

In relation to the performance of shared leadership, a number of key practices were identified. Information sharing and shared knowledge within the peer leaders' teams were deemed crucial to the effective running of the dialogue sessions. This involved group discussion when disagreements among team members arose, and the sharing of best practices with other peer leaders which allowed experienced team members to nurture and prepare the new ones.

"Sometimes we do disagree... when something happens in the group, we discuss it afterwards... we can have different views about the way of doing things" (Participant $4 \mathrm{MH}$ ) 
"We would talk about what went well, what did not go well. Sometimes amongst ourselves we would talk about specific things that have happened, like specific comments made" (Participant $6 \mathrm{MH}$ )

"Having an experienced person there so you can learn from their mistakes or hearing what they are doing, like do that or don't do that it helps so much." (Participant 4 TVA)

Such a sharing of knowledge and experiences also occurred between peer leaders and participants of dialogue groups and was seen as paramount to the intervention's success.

"Being able to share stories with people about things that've happened to you, us personally ... it made it hit home a little bit more. It's not just a story you hear on the video. [It] happened, you were there" (Participant $2 \mathrm{MH}$ )

"We were not giving [participants] information. We were chatting about scenarios, chatting about experiences, all learning together. I have learned loads... I feel that everyone in the room learns something" (Participant 3 TVA)

As the groups progressed, peer leaders were able to identify their own and their team-mates' strengths and weaknesses. Gradually, they settled into their roles and shared tasks according to their skills, making the dialogue sessions easier to run. Thus, skills pooling, task interdependence, shared responsibility and distributed power were identified in the data.

"[Running groups got easier] as people have settled into their roles. For instance, I know that on Thursday [more experienced AlcoLOLs] will take on the role of leading facilitator and that is fine with me. I ... don't like to be the main person that speaks all the time. It has been a lot easier" (Participant 8 TVA)

"As the facilitators got to know each other better [running groups] became lots easier as we became more comfortable with each other. We could interject with our own opinions with other people comfortably, without feeling it will be awkward or we were interrupting them. We all grew to work as a team while doing the facilitating which made the whole process a lot easier." (Participant 1 TVA)

"In AlcoLOLs you kind of have to work as a team. It is not something you can do by yourself... working together takes away the teacher figure... if it is like a group of people is spread evenly" (Group interview SFH)

Operating as facilitators led to the development of a collective identity based on shared values. This manifested in the data as references to the recognition of other members of the same collective and a recognition of the need to enact the shared values.

"We associate ourselves as a group if we see each other around the school. If I see someone that is a facilitator... it's like 'Oh, you are in the AlcoLOLs.'” (Participant 1 TVA) 
"When I'm out with [other AlcoLOLs], we always talk about it. ... [You] need to... practice what you preach." (Participant $6 \mathrm{MH}$ )

Team empowerment came about as a consequence of the role peer leaders played in the project. They felt they owned it, that they were in control.

"There is not a teacher, an adult in the room, this gives us an opportunity to take the lead. So we are not the ones talked at, we are the ones doing the talking." (Participant 6 TVA)

Consequently, peer leaders were not afraid to implement changes when they felt changes were needed, demonstrating the continuous development of Voice, as mentioned earlier on. The data show also that sustained effort and resilience were instrumental to the success of the project. Running multiple sessions reduced task ambiguity and improved performance.

"[Each year peer leaders] put their own twist in it... Last time I ran it, I had totally different experiences and totally different stories... Everybody has their own spin that they put on it." (Participant 3 TVA)

"Running groups is not as stressful as it used to be, the first groups were a bit stressful; we now know what we need to do, we got a flow that we follow..." (Participant $3 \mathrm{MH}$ )

\section{Outcomes}

The conceptual model of shared leadership presented earlier considers its outcomes as features of shared process, such as Team Performance, Task and Team Satisfaction. In our intervention, as highlighted in the previous section, participants believed that their team performance improved with the progression of the project, as they learned about their own and co-facilitators' skills, developed a clearer routine, and gained experience in dealing with the groups. Participants recognised that sharing responsibilities and working as team made the running and organisation of the sessions less demanding and improved their performance. Specifically, the interviews showed that participants derived task satisfaction and team satisfaction from the process. Helping other people enabled the AlcoLOLs to gain confidence and made them feel good about themselves and their involvement in the project. By working together, facilitators developed a bond and a sort of AlcoLOLs identity which according to them was instrumental to the success of the project.

"Sometimes you just need to be able to talk about things, to get them out in the open ... and it was really good to be part of the group that allowed that to happen." (Participant $2 \mathrm{MH}$ )

"If it wasn't that we [peer leaders] got on so well, I don't think it would work to be honest..." (Participant 2 TVA).

In our data, we also found evidence of individual level outcomes related to shared leadership, such as interpersonal and communication Skills Development, and changes in the way peer leaders behaved in situations involving alcohol. We see this ability to regulate one's own 
drinking behaviour as part of the development of new alcohol praxis combining new knowledge, critical reflection and communication skills.

"The AlcoLOLs breaks down barriers. Now I know how to talk to anybody - even groups who I wouldn't normally feel I could talk to." (Participant $2 \mathrm{SFH}$ )

"As facilitators we learned a lot about how we handle people ... we learned a lot about how to lead..." (Participant $2 \mathrm{MH}$ )

"[At the start, we thought] drinking wasn't really a big problem. And now I really do see it differently." (Participant 1 TVA)

"[The project] made me think differently about the whole party situation, hearing the stories from other people ... made me be slightly more responsible in my actions" (Participant $3 \mathrm{MH}$ )

In the context of the AlcoLOLs project, the occurrence and progression of Skills Development, Team Performance, Task and Team Satisfaction as well as Voice, Task Ambiguity, Task Cohesion, and Shared Values challenge the sequencing imposed on shared leadership by conventional conceptualisations as formulated in Figure 1. Rather than rigid placement within separate stages of antecedents, performance and outcomes of shared leadership, in our intervention there was an element of continuity between the three. Furthermore, connections we identified between practices of shared leadership, such as Sustained Effort, development of Shared Values and Collective Identity, as well as knowledge production and sharing suggest that the intervention has to run for a number of years to fully achieve its potential. To conclude, while it is important to distinguish between antecedents, process and outcomes, in the context of peer education, it is equally important to see shared leadership as an iterative rather than only a linear process.

\section{Conclusions}

Drawing from the discussion above, a model for a whole-school alcohol peer education intervention has been developed (see Figure 2 below).

The key initial step in this shared leadership model is the reframing of alcohol consumption: drinking/abstinence are not treated as behaviours but rather as actions, highlighting intentionality and choice involved. In addition, a collective, civic orientation is injected into individual's ways of acting and interacting in relation to alcohol. As de Vreede et al. argued, 'action taking requires that youth gain a broader skill set, including citizenship, teamwork, critical thinking skills [and] transformative learning' (2017:38). Transformative learning for the peer leaders in our project was enabled both by the group diversity and the developing sense of shared values and identity. Consequently, we can reframe 'peerness': rather than think of it as identification based on similarity and conformity, we see it as driven by aspiration and transformation. 
Figure 2: Shared leadership model of intervention

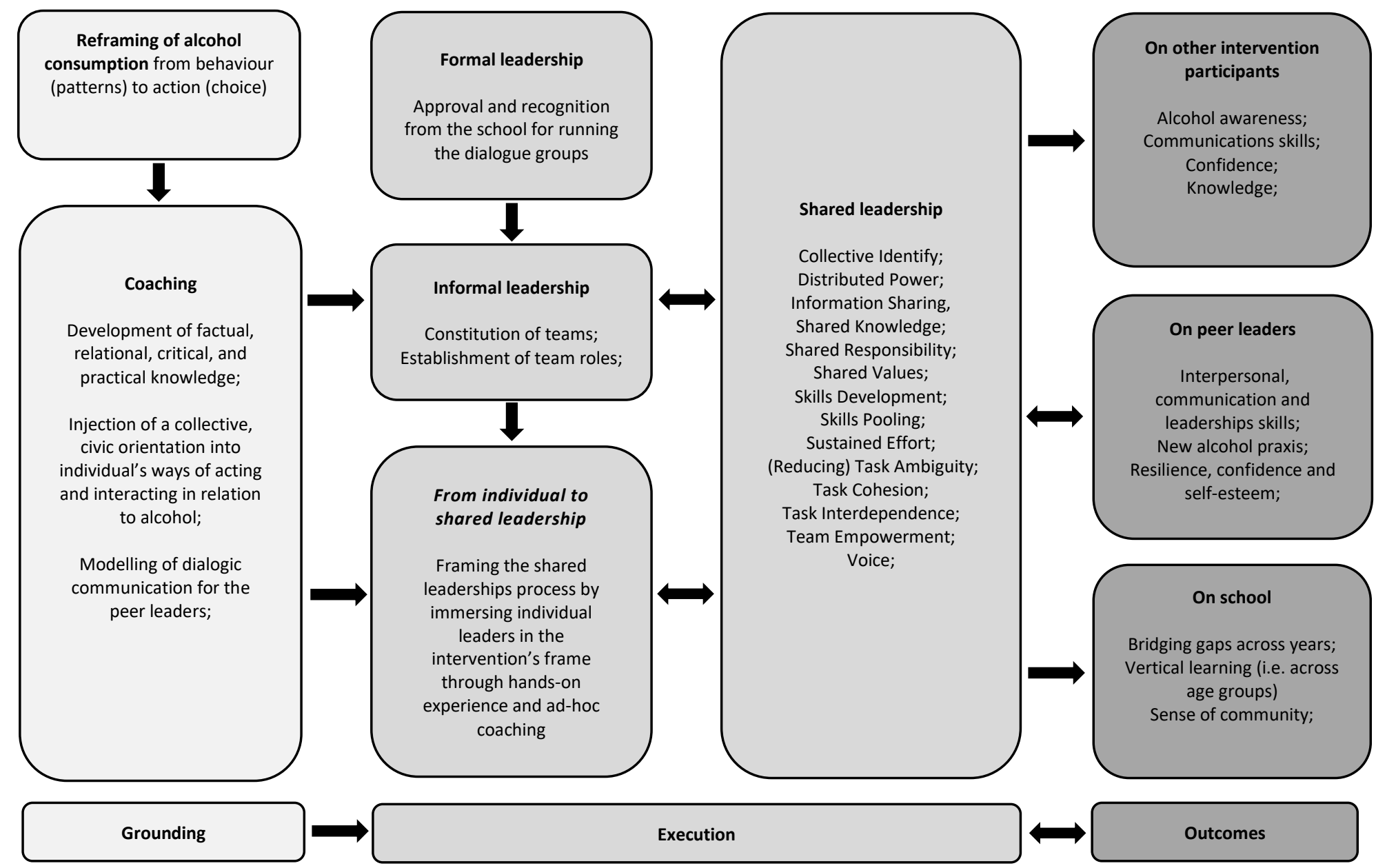

The emergence of the group dynamic that balanced diversity with similarity of shared goals and identity was, in turn, linked to the need to rely largely on informal leadership, on credibility of shared group performance in leading dialogue groups. This was rooted in the intervention's design and enhanced in the iterative process by: team empowerment, power distribution, skills pooling and development, as well as sharing of information, responsibility and values within the group. The intervention was thus premised on a more agentic and holistic view of young people as subjects in health interventions. While the focus in this article was on the group of peer leaders, the intervention as a whole produced outcomes at three different levels - on the peer educators themselves, on dialogue group participants and on schools as social systems. The outcomes on individuals and schools are discussed elsewhere (for more details see Pieczka et al. 2016).

To return to the questions we posed about peer leadership earlier, in our intervention leadership was shared rather than individual. Although amongst the group of 200 pupils who performed this role, there were individuals more able to command the room, to motivate, to envisage new ways and thus able to enact a traditional model of individual leadership, on the whole it was the pooling of skills, sharing of tasks and the ensemble performance that delivered leadership to the project. 


\section{Acknowledgements}

We thank The Robertson Trust, Portobello High School, Castlebrae Community High School, Drummond Community High School, Holy Rood RC High School, Leith Academy, Trinity Academy, all school staff, pupils, and peer leaders for making the AlcoLOLs project possible.

\section{Funding}

The AlcoLOLs project was funded by The Robertson Trust between 2013 to 2015 . 


\section{References}

Alcohol (Minimum Pricing) (Scotland) Act 2012). Available at: http://www.legislation.gov.uk/asp/2012/4/pdfs/asp 20120004 en.pdf (accessed 30 November 2012).

Barbour RS (1998) Mixing qualitative methods: quality assurance or qualitative quagmire? Qualitative Health Research 8(3): 352-361.

Borgia P, Marinacci C, Schifano P and Perucci CA (2005) Is peer education the best approach for HIV prevention in schools? Findings from a randomized controlled trial. Journal of Adolescent Health 36(6): 508-516.

Braun V and Clarke V (2006) Using thematic analysis in psychology. Qualitative Research in Psychology 3(2): 77-101.

Broome BJ (2009) Dialogue theories. In: Littlejohn SW and Foss KA (eds) Encyclopedia of Communication Theory Volume 1. Thousand Oaks: Sage Publications, pp. 301-306.

Carson JB, Tesluk PE and Marrone JA (2007) Shared leadership in teams: An investigation of antecedent conditions and performance. Academy of Management Journal 50(5): 1217-1234.

Cuijpers P (2002) Peer-led and adult-led school drug prevention: A meta-analytic comparison. Journal of Drug Education 32(2): 107-119.

Cullen-Lester CJ and Yammarino F (eds) (2016a) Collective and network approaches to leadership: Special issue introduction. The Leadership Quarterly 27(2): 173-348.

Cullen-Lester CJ and Yammarino F (2016b) Collective and network approaches to leadership: Special issue introduction. The Leadership Quarterly 27(2): 173-180.

de Visser RO, Graber R, Hart A, Abraham C, Memon A, Watten P and Scanlon T (2015) Using qualitative methods within a mixed-methods approach to developing and evaluating interventions to address harmful alcohol use among young people. Health Psychology 34 (4): 349-360.

de Visser RO, Wheeler Z, Abraham C and Smith J (2013) 'Drinking is our modern way of bonding': Young people's beliefs about interventions to encourage moderate drinking. Psychology and Health 28(12): 1460-1480.

de Vreede C, Warner A and Pitter R (2014) Facilitating Youth to take sustainability actions: The potential of peer education. The Journal of Environmental Education 45(1): 3756.

Denzin NK and Lincoln YS (2005) Qualitative Inquiry. 2nd ed. Thousand Oaks, CA: Sage.

Fisher WA, Fisher JD and Harman J (2003) The Information-Motivation-Behavioral Skills Model: A General Social Psychological Approach to Understanding and Promoting Health Behavior. In: Suls J and Wallston K A (eds) Social Psychological Foundations of Health and Illness, Blackwell Publishing, pp. 82-106.

Foxcroft D and Tsertsvadze A (2011) Universal school-based prevention programs for alcohol misuse in young people. Cochrane Database of Systematic Reviews 11(5): CD009113.

Gray L and Leyland AH (2017) Alcohol. Scottish Health Survey 2016: Volume 1: Main Report. Available at: https://www.gov.scot/publications/scottish-health-survey-2016volume-1-main-report/ (accessed 4 January 2019).

Harden A, Oakley A and Oliver S (2001) Peer-delivered health promotion for young people: a systematic review of different study designs. Health Education Journal 60(4): 339-353. 
Institute of Alcohol Studies (2018) Consumption of Alcohol Factsheet. Available at: http://www.ias.org.uk/Alcohol-knowledge-centre/Consumption/Factsheets/UKalcohol-consumption.aspx (accessed 20 March 2019).

Lambert SD and Loiselle CG (2008) Combining individual interviews and focus groups to enhance data richness. Journal of Advanced Nursing 62(2): 228-237.

Lee NK, Cameron J, Battams S, Roche A (2016) What works in school-based alcohol education: a systematic review. Health Education Journal 75(7): 780-798.

Livingstone AG, Young H and Manstead ASR (2011) "We Drink, Therefore We Are": The role of group identification and norms in sustaining and challenging heavy drinking "Culture". Group Processes \& Intergroup Relations 14(5): 637-649.

Mason-Jones AJ, Mathews C and Flisher AJ (2011) Can peer education make a difference? Evaluation of a South African adolescent peer education program to promote sexual and reproductive health. AIDS and Behavior 15(8): 1605-1611.

McDonald D (2004) Alcohol and Other Drug Peer Education in Schools: A review for the ACT Alcohol, Tobacco and Other Drug Strategy Implementation and Evaluation Group. Canberra: ACT Health Directorate.

McKeganey S (2000) The rise and rise of peer education approaches. Drugs: Education, Prevention and Policy 7(3): 293-310.

Namey E, Guest G, McKenna K and Chen M (2016) Evaluating bang for the buck: a costeffectiveness comparison between individual interviews and focus groups based on thematic saturation levels. American Journal of Evaluation 37(3): 425-440.

NHS Health Scotland (2016) Monitoring and Evaluating Scotland's Alcohol Strategy: Final Annual Report._Available at: http://www.healthscotland.scot/media/1100/mesasfinal-annual-report 5780 mar-2016.pdf (accessed 20 March 2019).

Ochieng B (2003) Adolescent health promotion: the value of being a peer leader in a health education/promotion peer education programme. Health Education Journal 62(1): 61-72.

Office for National Statistics (2017) Statistical bulletin: Adult drinking habits in Great Britain 2017. Available

at: https://www.ons.gov.uk/peoplepopulationandcommunity/healthandsocialcare/drug usealcoholandsmoking/bulletins/opinionsandlifestylesurveyadultdrinkinghabitsingre atbritain/2017\#in-great-britain-an-estimated-292-million-adults-drank-alcohol (accessed 4 January 2019).

Percy A, Wilson J, McCartan C and McCrystal P (2011) Teenage Drinking Cultures. Joseph Rowntree Foundation. Available at http://www.jrf.org.uk/sites/files/jrf/teenagedrinking-culture-full.pdf (accessed 30 November 2012).

Pieczka M (2011) Public relations as dialogic expertise?. Journal of Communication Management 15(2): 108-124.

Pieczka M and Wood E (2013) Action research and public relations: Dialogue, peer learning, and the issue of alcohol. Public Relations Inquiry 2(2): 161-181.

Pieczka M, Wood E and Casteltrione I (2016) The AlcoLOLs project: The Final Report. Queen Margaret University. Available at: https://eresearch.qmu.ac.uk/handle/20.500.12289/8988 (accessed 19 September 2018).

Pieczka M, Wood E and Escobar O (2010) Dialogue in Scotland?: A forum with communication practitioners. Working Paper. Centre for Dialogue: Queen Margaret University. Available at http://eresearch.qmu.ac.uk/2718/ (accessed 4 August 2019). 
Pearce CL and Conger JA (2003) Shared leadership: Reframing the Hows and Whys of Leadership. Thousand Oaks, CA: Sage Publications.

Public Health England (2016) The Public Health Burden of Alcohol and the Effectiveness and Cost-Effectiveness of Alcohol Control Policies: An Evidence Review. Available at: https://assets.publishing.service.gov.uk/government/uploads/system/uploads/attac hment data/file/733108/alcohol public health burden evidence review update 2018.pdf (accessed 19 September 2018).

Robson C (2011) Real World Research. 3rd ed. New York: John Wiley \& Sons.

Sacks-Davis R, Horyniak D, Grebely J and Hellard M (2012) Behavioural interventions for preventing hepatitis $C$ infection in people who inject drugs: A global systematic review. International Journal of Drug Policy 23(3): 176-184.

Scottish Executive (2002) Plan for Action on Alcohol Problems. Available at: http://alcoholresearchuk.org/wp-content/uploads/2014/01/scottish-executive-planfor-action-2002.pdf (accessed 30 November 2012).

Serban A and Roberts A (2016) Exploring antecedents and outcomes of shared leadership in a creative context: A mixed-methods approach. The Leadership Quarterly 27(2): 181199.

Shiner M (1999) Defining peer education. Journal of Adolescence 22(4): 555-566.

Smith L and Foxcroft D (2009) Drinking in the UK: An Exploration of Trends. Joseph Rowntree Foundation. Available at: https://www.jrf.org.uk/sites/default/files/jrf/migrated/files/UK-alcohol-trendsFULL.pdf (accessed 19 September 2018).

Southgate $E$ and Aggleton P (2017) Peer education: From enduring problematics to pedagogical potential. Health Education Journal 76(1): 3-14.

Stokes D and Bergin R (2006) Methodology or methodolatry? An evaluation of focus groups and depth interviews. Qualitative Market Research: An International Journal 9(1): 2637.

Tobin GA and Begley CM (2004) Methodological rigour within a qualitative framework. Journal of Advanced Nursing 48(4): 388-396.

Tolli MV (2012) Effectiveness of peer education interventions for HIV prevention, adolescent pregnancy prevention and sexual health promotion for young people: A systematic review of European studies. Health Education Research 27(5): 904-913.

Velleman, R (2009) Children, Young People and Alcohol: How They Learn and How to Prevent Excessive Use. Joseph Rowntree Foundation. Available at http://www.jrf.org.uk/publications/young-people-alcohol- excessive-prevention (accessed 30 November 2012).

Webel AR, Okonsky J, Trompeta J and Holzemer WL (2010) A systematic review of the effectiveness of peer-based interventions on health-related behaviors in adults. American Journal of Public Health 100(2): 247-253.

White L, Currie G and Lockett A (2016) Pluralized leadership in complex organizations: Exploring the cross-network effects between formal and informal leadership relations. The Leadership Quarterly 27(2): 280-297.

World Health Organization (2019) Heavy episodic drinking among drinkers. Global Information System on Alcohol and Health (GISAH): Patterns of consumption. Available at:

https://www.who.int/gho/alcohol/consumption patterns/heavy episodic drinkers text/en/ (accessed 4 January 2019). 
Wierzbicka A (2006) The concept of dialogue in cross-linguistic and cross-cultural perspective. Discourse Studies 8(5): 675-703.

Wyborn I (2016) Youth drinking in transition. London: Youth drinking in transition. London: Demos. Available at:

https://www.demos.co.uk/wpcontent/uploads/2016/09/Youth-drinking-web.pdf (accessed 19 September 2018).

Yammarino, F, Salas E, Serban A, Shirreffs K, and Shuffler M (2012) Collectivistic leadership approaches: Putting the "we" in leadership science and practice. Industrial and Organizational Psychology 5(4): 382-402. 